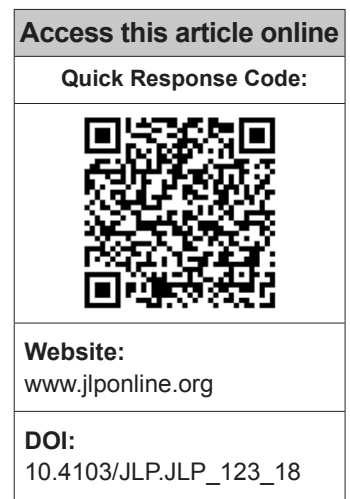

Departments of Microbiology and ${ }^{1} P$ aediatrics, King George's Medical University, ${ }^{2}$ Department of Community Medicine,

Integral Institute of Medical Sciences and Research, ${ }^{3}$ Department of Microbiology, Sanjay

Gandhi Postgraduate Institute of Medical Sciences, ${ }^{5}$ Department of Microbiology, Era's Lucknow Medical College and Hospital, Lucknow,

Uttar Pradesh,

${ }^{4}$ Department of

Microbiology, All India

Institute of Medical

Sciences, Bhopal,

Madhya Pradesh, India

Address for correspondence:

Dr. Sheetal Verma,

Department of Microbiology, King George's Medical University, Chowk,

Lucknow - 226003 , Uttar Pradesh, India. E-mail: dr.sheetal2001@ gmail.com

Submission: 25-09-2018 Accepted: 15-12-2018

\title{
Etiological agents of diarrhea in hospitalized pediatric patients with special emphasis on diarrheagenic Escherichia coli in North India
}

\author{
Sheetal Verma, Vimala Venkatesh, Rashmi Kumar', Saurabh Kashyap², \\ Manoj Kumar ${ }^{3}$, Anand Kumar Maurya ${ }^{4}$, T. N. Dhole ${ }^{3}$, Mastan Singh ${ }^{5}$
}

\begin{abstract}
:
INTRODUCTION: Infectious diarrhea is leading infectious cause of childhood morbidity, hospitalizations, and mortality particularly in children living in developing countries like India. The etiological agents differ depending on geographical area, and recent data suggest increase in drug resistance to various enteropathogens.
\end{abstract}

AIMS AND OBJECTIVES: The aim of the study was to investigate emerging diarrheal agents and antimicrobial resistance profile of bacterial pathogens from children ( $<12$ years of age) hospitalized with acute diarrhea.

MATERIALS AND METHODS: A cross-sectional, hospital-based observational study was conducted over 1 year in which 100 children $<12$ years who were hospitalized due to diarrhea were recruited. Diarrhea was defined as the passage of three or more liquid stools in a 24-h period using the World Health Organization guidelines. Samples were processed for detection of various bacterial, viral, and parasitic agents by standard microbiological, serological, and molecular tests. Antimicrobial resistance testing was performed with the Kirby-Bauer disk diffusion method. ELISA was performed for Rotavirus and Escherichia coli O157. Multiplex polymerase chain reaction test was performed to detect diarrheagenic E. coli (DEC).

RESULTS: Pathogenic diarrheal agents were found in $63 \%$ patients. Rotavirus was identified in $52.5 \%$, DEC in 29\%, Vibrio cholerae in 4\%, Shigella flexneri in 3\%, Aeromonas sp. in 1\%, Giardia lamblia in $4 \%$, and Entamoeba histolytica in 1\% cases. Enteropathogenic E. coli (EPEC) in $19(65.5 \%)$ cases was the most common agent followed by Enteroaggregative E. coli (EAEC) in $5(17.2 \%)$, Enterotoxigenic E. coli (ETEC) in 2 (6\%), and Enteroinvasive E. coli (EIEC) in $3(10.3 \%)$ cases. Resistance rates of DEC to first-line therapeutic drugs were high, $97.3 \%$ to ampicillin and $95.95 \%$ to co-trimoxazole. DEC was susceptible to chloramphenicol in $58.11 \%$, gentamicin in $48.19 \%$, and amikacin in $58.11 \%$ cases. Shigella sp. and V. cholerae isolates were $100 \%$ sensitive to gentamicin and ofloxacin.

CONCLUSION: EPEC is the most common DEC pathotype and EAEC, ETEC, and EIEC are also emerging as dominant diarrheal agents. Rotavirus was the most common causative agents of diarrhea especially in children $<5$ years. Most of the bacterial isolates showed high level of drug resistance to first-line empirical drugs and were multidrug resistant making them unsuitable for empiric treatment. Laboratory monitoring of drug susceptibility of stool isolates appears necessary to formulate antibiotic policy for treating diarrheal illness at the local level. There is an urgent need to strengthen diarrheal surveillance to monitor susceptibility to commonly prescribed antibiotics.

Key words:

Diarrhea, diarrheagenic Escherichia coli, drug resistance, enteropathogens, rotavirus

This is an open access journal, and articles are distributed under the terms of the Creative Commons Attribution-NonCommercial-ShareAlike 4.0 License, which allows others to remix, tweak, and build upon the work non-commercially, as long as appropriate credit is given and the new creations are licensed under the identical terms.

For reprints contact: reprints@medknow.com
How to cite this article: Verma S, Venkatesh V, Kumar R, Kashyap S, Kumar M, Maurya AK, et al. Etiological agents of diarrhea in hospitalized pediatric patients with special emphasis on diarrheagenic Escherichia coli in North India. J Lab Physicians 2019;11:68-74. 


\section{Introduction}

D iarrheal disease ranks among "three giant killers" of infants and children. Each year globally, 1.7 billion cases of childhood diarrheal disease are reported with 525,000 deaths in children under five..$^{[1]}$ Nearly half of these diarrheal cases are reported from South Asia and Africa and cause morbidity, mortality, and other severe outcomes. ${ }^{[2]}$ India alone is responsible for estimated $300,000(13 \%)$ of all deaths per year in children under 5 years of age due to diarrhea. ${ }^{[3]}$ In India, about one-third of hospital admissions are due to diarrheal diseases and $17 \%$ of all deaths in hospitalized patients are diarrhea related. ${ }^{[4]}$ Lack of education, poor quality potable water, limited sanitation, poor hygiene practices, and socioeconomic factors make the situation worse for diarrhea control in developing countries. ${ }^{[5]}$

The majority of diarrheal diseases among the children in developing countries are caused by infectious etiological agents such as Rotavirus, diarrheagenic Escherichia coli (DEC), Shigella sp., Salmonella sp., and Entamoeba histolytica. ${ }^{[6]}$ The five categories of DEC usually encountered are Enteropathogenic E. coli (EPEC), Enterohemorrhagic E. coli (EHEC), Enterotoxigenic E. coli (ETEC), Enteroaggregative E. coli (EAEC), and Enteroinvasive E. coli (EIEC). ${ }^{[7]}$ The gold standard tests for the definitive diagnosis of bacterial pathogens' infection currently depend on cultivation of the pathogenic organisms, which requires up to 3 days for final identification by standard biochemical tests. The pathogen yield from samples decreases if it is not fresh, delay in transportation, patient on prior antibiotics, or inappropriate sample collection. The antimicrobial resistance is an overgrowing problem worldwide, and there is an urgent need to monitor the susceptibility pattern of common bacterial isolates for drugs used in diarrheal disease to formulate guidelines for the empirical treatment. ${ }^{[8]}$

Early diagnosis of the infectious agent causing diarrhea is extremely essential especially in young children for proper management to reduce morbidity and mortality. Identification of diarrheagenic causing pathogens including bacterial, viral, and parasites is necessary for policymaking decision at the local and national level. Various studies have found that enzyme immunoassay and molecular methods may increase the detection rate compared to conventional methods. ${ }^{[9]}$ The information on DEC including E. coli O157: $\mathrm{H7}$ as an etiological agent of diarrhea in Indian children is scarce. Pal et al., ${ }^{[10]}$ first reported EHEC from nondiarrheagenic animal sources in India. Various studies from animals, food, and humans thereafter have suggested that this enteropathogen may be a human health problem in future. ${ }^{[11]}$ To provide more insights into the etiology of acute diarrhea with special emphasis on DEC in North India, this study was conducted in children below 12 years hospitalized due to diarrhea.

\section{Materials and Methods}

\section{Clinical definitions and study population}

A cross-sectional, hospital-based observational study was conducted over a period of 1 year in children $<12$ years admitted with acute diarrhea in a university hospital. In India, studies have determined isolation of enteropathogens to be from $8 \%$ to $12 \%$. A sample size of 100 was calculated, according to OpenEpi, Version 3 (Bill and Melinda Gates Foundation, Andrew G. Dean, Kevin M. Sullivan, and Roger Mir, Atlanta, Georgia), $n=($ DEFF * Np [1-p] $) /\left(\left[d 2 / Z 21-\alpha / 2^{*}[N-1]+p^{*}[1-p]\right)\right.$. Hypothesized \% frequency of outcome factor in the population (p):10.3\% $\pm 5 \%$ at a confidence limit of $90 \%$.

Diarrhea was defined using the World Health Organization guidelines criteria as passage of three or more liquid stools in a 24-h period. Children having diarrhea more than 14 days and who received antibiotics before admission were excluded from the present study. Stool samples from acute diarrheal children were collected after parents or guardian's permission. Demographic information for each patient such as age, sex, and clinical symptoms were collected on a structured questionnaire.

Sample collection and identification of pathogens

5-10 $\mathrm{ml}$ of freshly passed single stool sample or stool sample from diaper were collected in a clean, dry, and leak-proof wide mouth plastic container and transported to the laboratory within $2 \mathrm{~h}$. Specimen from hospital pan and rectal swabs were not collected. Samples were processed immediately for microscopy and culture. An aliquot of about $0.5 \mathrm{ml}$ was refrigerated at $-20^{\circ} \mathrm{C}$ for subsequent ELISA and polymerase chain reaction (PCR) testing.

The stool samples were examined grossly, direct wet mount with normal saline $(0.85 \% \mathrm{NaCl})$ solution and by modified Ziehl-Neelsen method. ${ }^{[12]}$ Stool samples were inoculated on MacConkey agar (Hi-Media, Mumbai), Xylose lysine deoxycholate agar (Hi-Media, Mumbai), and thiosulfate bile salt agar (Hi-Media, Mumbai). Morphological characteristics of colonies were examined after overnight incubation at $37^{\circ} \mathrm{C}$ for $18-24 \mathrm{~h}$, and standard biochemical tests were performed. Screening for E. coli O157 was done on Sorbitol MacConkey (SMAC) agar (Hi-Media, Mumbai), which was prepared as described previously. ${ }^{[13]}$ Dehydrated MacConkey Agar Base was obtained from Accumix, Microxpress (Tulip Diagnostics Ltd, Goa, India), and SMAC agar was prepared in-house by adding 1\% D-Sorbitol (Hi-Media, Mumbai, India). Sorbitol nonfermenting colonies were 
picked and further processed by standard biochemical tests. Bacterial isolates obtained from pure culture were lyophilized in two ampoules and stored for future testing.

\section{Serotyping}

EHEC O157: H7 diagnosis was carried out by slide agglutination test using commercially available O157 and H7 antisera (IVD-Denka Seiken Co., Ltd, Tokyo, Japan). The serotyping of Shigella spp., Vibrio spp., and Salmonella spp. were performed by slide agglutination test using specific antisera.

\section{Antimicrobial sensitivity test}

The antimicrobial susceptibility profile of the isolates was determined by Kirby-Bauer disk diffusion method using different antimicrobial agent (Hi-Media, Mumbai, India) according to the guidelines recommended by Clinical and Laboratory Standards Institute. ${ }^{[14]}$ All isolates recovered from culture were tested with ampicillin $(10 \mu \mathrm{g})$, cefotaxime $(30 \mu \mathrm{g})$, ceftriaxone $(30 \mu \mathrm{g})$, cotrimoxazole $(25 \mu \mathrm{g})$, tetracycline $(30 \mu \mathrm{g})$, doxycycline $(10 \mu \mathrm{g})$, chloramphenicol $(30 \mu \mathrm{g})$, ciprofloxacin $(5 \mu \mathrm{g})$, nalidixic acid $(30 \mu \mathrm{g})$, norfloxacin $(10 \mu \mathrm{g})$, ofloxacin $(5 \mu \mathrm{g})$, azithromycin $(15 \mu \mathrm{g})$, gentamicin $(10 \mu \mathrm{g})$, amikacin $(30 \mu \mathrm{g})$, cefixime $(5 \mu \mathrm{g})$, cefuroxime $(30 \mu \mathrm{g})$, cefepime $(30 \mu \mathrm{g})$, ceftazidime $(30 \mu \mathrm{g})$, cefoperazone/sulbactam $(75 / 30 \mu \mathrm{g})$, piperacillin/tazobactam $(100 / 10 \mu \mathrm{g})$, and imipenem $(10 \mu \mathrm{g})$. Multidrug resistance was defined as resistant to $\geq 3$ antimicrobial categories. E. coli ATCC 25922 and Staphylococcus aureus ATCC 25923 were chosen as quality control strains.

\section{ELISA testing}

The ELISA for E. coli O157:H7 was performed by commercial available kit E. coli O157 Antigen Detection Microwell ELISA (ECO-96, IVD Research Inc, USA) in all the stool samples. The ELISA for Rotavirus was performed by commercially available kit
RIDASCREEN Rotavirus (C0901) Enzyme immunoassay for detection of Rotavirus in the stool (R-Biopharm AG, Darmstadt, Germany).

\section{Molecular diagnostic methods for diarrheagenic} Escherichia coli

DEC was characterized by PCR as previously described. [15] EPEC (eaeA and bfpA), STEC (stx1 and stx2), ETEC (elt), EIEC (ipaH), and EAEC (CVD432) were detected by two multiplex PCR assays. First multiplex PCR assay utilized three primer pairs and detected the presence of eae, bfpA, and the target of CVD432. Second multiplex PCR assay used stx1, stx2, elt, and ipaH [Table 1]. Boiling method was used for DNA extraction; bacterial strains were cultured on MacConkey agar and revived from lyophilized vials stored. A sweep of about five E. coli-like colonies were suspended in $50 \mu \mathrm{l}$ of deionized water, boiling the suspension for $10 \mathrm{~min}$ at $95^{\circ} \mathrm{C}$, and centrifuging it at 10,000 $\times \mathrm{g}$ for $10 \mathrm{~min}$. The supernatant was used as the DNA template for PCR. The optimized protocol was carried out with a PCR reaction mixture (50 $\mu 1)$ containing $10 \mathrm{mM}$ Tris- $\mathrm{HCl}(\mathrm{pH} 8.3) ; 50 \mathrm{mM} \mathrm{KCl}$; $0.1 \%$ Triton X-100; $1.5 \mathrm{mM} \mathrm{MgCl2} 2.5 \mathrm{U}$ of Taq DNA polymerase; 10 pmole of each primer (SBS Gentech Co. Ltd., India); and $5 \mu$ l of extracted DNA. The PCR mixtures were then subjected to the following cycling conditions: For assay $1,50^{\circ} \mathrm{C}(2 \mathrm{~min}, 1$ cycle $) ; 95^{\circ} \mathrm{C}$ (5 min, 1 cycle); 40 cycles of $95^{\circ} \mathrm{C}(40 \mathrm{~s}), 58^{\circ} \mathrm{C}(1 \mathrm{~min})$, and $72^{\circ} \mathrm{C}(2 \mathrm{~min})$; and a final extension step at $72^{\circ} \mathrm{C}$ (7 min, 1 cycle); and for assay $2,50^{\circ} \mathrm{C}\left(2 \mathrm{~min}, 1\right.$ cycle); $95^{\circ} \mathrm{C}(5 \mathrm{~min}$, 1 cycle); 40 cycles of $95^{\circ} \mathrm{C}(45 \mathrm{~s}), 50^{\circ} \mathrm{C}(1 \mathrm{~min})$, and $72^{\circ} \mathrm{C}(1 \mathrm{~min})$; and $72^{\circ} \mathrm{C}$ (7 min, 1 cycle) in a thermal cycler (ABI 9700 GeneAmp Thermal Cycler). The amplified products were separated on $2 \%$ agarose gels, visualized on an ultraviolet-light transilluminator (Bio-Rad Laboratories). Local isolates of E. coli positive for above genes were used for standardization of the multiplex PCR assays. E. coli DH5 $\alpha$, which lacks all the diarrheagenic genes, was used as a negative control.

Table 1: Primer sequences and predicted lengths of polymerase chain reaction amplification products

\begin{tabular}{|c|c|c|c|c|c|}
\hline Strain & Target gene & Direction & Primer sequence $\left(5^{\prime}-3^{\prime}\right)$ & Fragment size (bases) & Reference \\
\hline \multirow[t]{4}{*}{ EPEC } & eaeA & Forward & TCAATGCAGTTCCGTTATCAGTT & 482 & Hegde et al., 2012[16] \\
\hline & & Reverse & GTAAAGTCCGTTACCCCAACCTG & & \\
\hline & bfpA & Forward & GGAAGTCAAATTCATGGGGGTAT & 300 & Hegde et al., 2012[16] \\
\hline & & Reverse & GGAATCAGACGCAGACTGGTAGT & & \\
\hline \multirow[t]{4}{*}{ EHEC } & stx1 & Forward & CAGTTAATGTGGTGGCGAAGG & 348 & Hegde et al., 2012[16] \\
\hline & & Reverse & CACCAGACAATGTAACCGCTG & & \\
\hline & stx2 & Forward & ATCCTATTCCCGGGAGTTTACG & 584 & Hegde et al., 2012[16] \\
\hline & & Reverse & GCGTCATCGTATACACAGGAGC & & \\
\hline \multirow[t]{2}{*}{ ETEC } & elt & Forward & TCTCTATGTGCATACGGAGC & 273 & Hegde et al., 2012[16] \\
\hline & & Reverse & TGGTCTCGGTCAGATATGTG & & \\
\hline \multirow[t]{2}{*}{ EIEC } & ipaH & Forward & GACGGACAACAGAATACACTCCATC & 108 & Hegde et al., 2012[16] \\
\hline & & Reverse & ATGTTCAAAAGCATGCCATATCTGT & & \\
\hline \multirow[t]{2}{*}{ EAEC } & CVD432 & Forward & CTGGCGAAAGACTGTATCAT & 630 & Hegde et al., 2012[16] \\
\hline & & Reverse & AAATGTATAGAAATCCGCTGTT & & \\
\hline
\end{tabular}




\section{Statistical analysis}

Using the SPSS software 20 (IBM, Armonk, NY, United States of America), the Chi-squared test was employed to determine the statistical significance of data. $P<0.05$ was considered as statistically significant.

\section{Results}

In the present study, out of 100 children, $66 \%$ were males and $34 \%$ were females, respectively. Majority of children, i.e., $43 \%$ were $<1$ year of age, $29 \%$ were between 12 and 36 months, 17\% were between 36 and 60 months, and $11 \%$ were above 60 months, respectively. Most patients were admitted in the summer season $(60 \%)$, followed by autumn $(21 \%)$, spring $(14 \%)$, and winter (5\%). About $82 \%$ of the admitted children with acute diarrhea showed fever, $73.9 \%$ had abdominal pain, and $69 \%$ complained vomiting [Table 2].

Cultures showed growth of E. coli in 92 cases, Shigella flexneri in three cases, Vibrio cholerae in four cases, and Aeromonas sp. in one case [Table 3]. No E. coli O157:H7 was recovered from SMAC agar. The antibiotic susceptibility pattern of the enteropathogenic isolates is shown in Table 4. Among the diarrheagenic parasitic agents, Giardia lamblia was seen in four cases and E. histolytica in one case. Cryptosporidium sp. was not found in any diarrheal stool sample. Rotavirus ELISA was performed in stool samples in children under 5 years, and it was found positive in $52.5 \%(21 / 40)$ samples tested. Majority of cases of Rotavirus, i.e., 14/21 (66.67\%) were between 6 and 12 months of age. One sample was positive for E. coli O157 antigen in stool on testing with ELISA. Among 100 collected stool samples, 29 cases were positive for DEC (overall prevalence 29\%). The most frequent pathotype of DEC was EPEC 19 (65.5\%), followed by EAEC 5 (17.2\%), ETEC 2 (6\%), EIEC $3(10.3 \%)$, and no STEC was found. In $63 \%$ of samples, definite known pathogenic etiological agent of diarrhea was found (DEC in 29, Rotavirus in 21, S. flexneri in three, $V$. cholerae in four, Aeromonas sp. in one, G. lamblia in four, and E. histolytica in one case). 38 children had single infection, 12 children had two types of infections, and 3 had more than 2 types of infections. In remaining $37 \%$ of samples, no definite etiological agent was found.

\section{Discussion}

Diarrhea is the most important cause of morbidity and mortality in young children in developing countries. In the present study, 100 children with diarrhea were included, and it was found that $43 \%$ patients were below 1 year with male preponderance which is similar to findings of other studies. ${ }^{[7,17,18]}$ In this study, overall detection of rotavirus was $52.5 \%$ in children under 5 years of age which is much higher than a similar
Table 2: Basic information and clinical symptoms of the study population $(n=100)$

\begin{tabular}{l|c|c}
\hline Characteristics & Total $(n)$ & Number of cases (\%) \\
\hline Sex & & \\
$\quad$ Male & 100 & $66(66)$ \\
Female & 100 & $34(34)$ \\
Age (years) & & \\
$<1$ & 100 & $43(43)$ \\
\hline $1-3$ & 100 & $29(29)$ \\
$3-5$ & 100 & $17(17)$ \\
$>5$ & 100 & $11(11)$ \\
Clinical symptoms & & \\
Fever & 100 & $82(82)$ \\
Vomiting & 100 & $69(69)$ \\
Abdominal pain & 23 & $17(73.9)$ \\
Tenesmus & 23 & $6(26.1)$ \\
Headache & 23 & $6(26.1)$ \\
Myalgia & 23 & $6(26.1)$ \\
Low urine output & 100 & $36(36)$ \\
Altered sensorium & 100 & $16(16)$ \\
Dehydration & & \\
Nil & 100 & $3(3)$ \\
Mild & 100 & $16(16)$ \\
Moderate & 100 & $49(49)$ \\
Severe & 100 & $32(32)$ \\
\hline
\end{tabular}

Table 3: Pattern of enteropathogens in stool in the study population $(n=100)$

\begin{tabular}{lc}
\hline Etiological agents & $\boldsymbol{n}(\%)$ \\
\hline Diarrheagenic Escherichia coli & $29(29)$ \\
EPEC & $18(18)$ \\
EAEC & $5(5)$ \\
ETEC & $2(2)$ \\
EIEC & $3(3)$ \\
EHEC & $1(1)$ \\
Shigella flexneri & $3(3)$ \\
Vibrio cholerae serogroup O1 & $4(4)$ \\
Aeromonas spp. & $1(1)$ \\
Entamoeba histolytica & $1(1)$ \\
Giardia lamblia & $4(4)$ \\
Ascaris lumbricoides & $1(1)$ \\
Enterobius vermicularis & $1(1)$ \\
Rotavirus & $21 / 40(52.5)$ \\
\hline
\end{tabular}

EPEC $=$ Enteropathogenic Escherichia coli, EAEC $=$ Enteroaggregative Escherichia coli, ETEC = Enterotoxigenic Escherichia coli,

$\mathrm{EIEC}=$ Enteroinvasive Escherichia coli, EHEC = Enterohemorrhagic Escherichia coli

study ${ }^{[19]}$ which reported $35 \%$ stool positivity for rotavirus antigen by ELISA. Data from 22 Indian cities were analyzed; a total of 15,476 samples were tested by various tests and rates of rotavirus positivity ranged from $6 \%$ to $45 \%{ }^{[20]}$ The DEC overall prevalence was $29 \%$. The most frequent pathotype was EPEC $19(65.5 \%)$, followed by EAEC $5(17.2 \%)$, ETEC $2(6 \%)$, EIEC $3(10.3 \%)$, and no STEC was found. In another study conducted in 200 children with diarrhea, DEC infections were found in $26 \%$; EAEC was the most common DEC identified by 
Verma, et al:: Characterization of diarrheagenic agents

Table 4: Bacterial enteropathogens in stool and their resistance pattern in percentage

\begin{tabular}{|c|c|c|c|c|}
\hline Antibiotic & Escherichia coli $(n=92)$ & Shigella flexneri $(n=3)$ & Vibrio cholerae $(n=4)$ & Aeromonas spp. $(n=1)$ \\
\hline Ampicillin & 97.30 & 100.00 & 50.00 & 100.00 \\
\hline Cefotaxime & 95.95 & 66.67 & 50.00 & 100.00 \\
\hline Ceftriaxone & 95.95 & 66.67 & 50.00 & 100.00 \\
\hline Cefixime & 95.95 & 66.67 & 50.00 & 100.00 \\
\hline Cotrimoxazole & 91.89 & 100.00 & 100.00 & 100.00 \\
\hline Tetracycline & 97.30 & 100.00 & 50.00 & 100.00 \\
\hline Doxycycline & 78.20 & 66.67 & 50.00 & 100.00 \\
\hline Azithromycin & 74.32 & 66.67 & 50.00 & 100.00 \\
\hline Chloramphenicol & 58.11 & 66.67 & 50.00 & 100.00 \\
\hline Ciprofloxacin & 93.24 & 100.00 & 50.00 & 0.00 \\
\hline Nalidixic acid & 100.00 & 100.00 & 0.00 & 0.00 \\
\hline Norfloxacin & 91.89 & 100.00 & 50.00 & 100.00 \\
\hline Ofloxacin & 66.79 & 0.00 & 0.00 & 0.00 \\
\hline Gentamicin & 58.11 & 0.00 & 0.00 & 0.00 \\
\hline Amikacin & 41.89 & 100.00 & 50.00 & 100.00 \\
\hline Cefuroxime & 97.30 & 66.67 & 50.00 & 100.00 \\
\hline Cefepime & 93.24 & 66.67 & 50.00 & 100.00 \\
\hline Ceftazidime & 90.54 & 66.67 & 50.00 & 100.00 \\
\hline Cefoperazone/sulbactam & 74.32 & 0.00 & 0.00 & 100.00 \\
\hline Piperacillin/tazobactam & 94.59 & 100.00 & 50.00 & 100.00 \\
\hline Imipenem & 50.00 & 66.67 & 50.00 & 100.00 \\
\hline
\end{tabular}

multiplex PCR followed by EPEC in 16\% cases, ETEC in $3.5 \%$, and EIEC in $1.5 \%$ of the diarrheal cases. ${ }^{[16]}$ Other similar studies have reported the incidence of $E$. coli to be $27.6 \%,{ }^{[21]} 43 \%{ }^{[7]}$ and $61.76 \%{ }^{[22]}$ respectively. The multiplex PCR assays are highly sensitive and useful for identification of DEC. ${ }^{[23]}$

In this study, resistance rates of DEC to first-line therapeutic drugs were high, for example, $97.3 \%$ to ampicillin and $95.95 \%$ to co-trimoxazole higher than rates reported before ${ }^{[24,25]}$ but appears similar to the study by Karmali ${ }^{[7]}$ in which only $9.30 \%$ E. coli strains were susceptible. In this study, $89 \%$ of E. coli isolates were multidrug resistant, which is much higher compared to other studies which reported $66.6 \%{ }^{[24]}$ and $70.2 \%$. ${ }^{[26]}$ The resistance was $100 \%$ for amoxicillin clavulanate and nalidixic acid, $97.3 \%$ for tetracycline, $93.24 \%$ for ciprofloxacin, and $97.3 \%$ for ampicillin. The E. coli isolates were susceptible to chloramphenicol (58.11\%), gentamicin $(48.19 \%)$, amikacin (58.11\%), and imipenem (50\%), respectively. The findings are similar to another study in hospitalized Indian children with diarrhea in which nalidixic acid was found to be $100 \%$ resistant and fluoroquinolone susceptibility was $13.95 \%$ among the DEC strains and in vitro sensitivity to amikacin was $83.72 \% .{ }^{[7]}$ Uma et al. ${ }^{[22]}$ found that $90 \%$ of $E$. coli strains were resistant to most of the antimicrobial agents tested; all the isolates were resistant to ampicillin, imipenem, and cotrimoxazole but were sensitive to amikacin.

About $66.67 \%$ of isolates of Shigella sp. in our study were resistant to ceftriaxone, cefixime, and azithromycin which are first-line drugs. Strains were also resistant to nalidixic acid, cotrimoxazole, furazolidone, ciprofloxacin, and ampicillin but were sensitive to gentamicin and ofloxacin. In a similar study, among Shigella, an overall resistance of $63.6 \%, 58.1 \%, 25.92 \%$, and $16.3 \%$ were observed for nalidixic acid, cotrimoxazole, ciprofloxacin, and furazolidone, respectively. ${ }^{[18]}$ Ciprofloxacin resistance though uncommon in Shigella has also been reported from other parts of India. ${ }^{[27]}$

In this study, $4 \%$ of isolates obtained were $V$. cholerae O1 subtype Ogawa. The other studies in India have also found subtype Ogawa infection to be more prevalent. ${ }^{[18]} V$. cholerae isolates showed resistance to doxycycline, azithromycin, and ciprofloxacin which are first-line drugs for treatment. Strains were susceptible to gentamicin but were resistant to nalidixic acid, cotrimoxazole, and furazolidone. Two isolates were susceptible to tetracycline, amoxicillin clavulanate, and ampicillin. Various studies have reported increase in resistance to ampicillin, cotrimoxazole, furazolidone, and nalidixic acid; complete resistance to furazolidone but susceptibility to gentamicin and tetracycline in $V$. cholerae isolates. ${ }^{[18,28]}$

All nonbloody stools submitted for the examination of bacterial enteric pathogens should be cultured for E. coli O157:H7.[29] The agar medium most commonly used for the isolation of E. coli O157:H7 is SMAC which is $100 \%$ sensitive, $85 \%$ specific, and $86 \%$ accurate for detecting E. coli O157:H7.[13] In this study, no E. coli O157:H7 was isolated by culture on SMAC agar. Our findings are also similar to many previous studies in India which 
found nil to rare isolates of E. coli O157.[7,11] In the present study, an overall detection rate by ELISA was 1/100 (1\%) from the study participants. Earlier studies reported cross-reaction of E. coli O157 lipopolysaccharide with antibodies to many other pathogens. ${ }^{[30]}$ E. coli O157:H7 in the present study was not detected by culture and PCR.

G. lamblia was observed in four (4\%) cases and E. histolytica in one $(1 \%)$ case which are known diarrhea-causing agents. In India, reports on the prevalence of giardiasis in children range from $2.6 \%$ to $12 \%{ }^{[31]}$ The presence of Ascaris lumbricoides and Enterobius vermicularis in one sample each appears to be of uncertain significance. Cryptosporidium sp. was not detected in the present study. In India, reports that are available indicate a prevalence rate of Cryptosporidium between $1 \%$ and $16.7 \%$. $^{[32]}$ The pathogenic etiological agents in the present study were found in $63 \%$ cases. In various studies, pathogens were detected varying from $10 \%$ to $70 \%$ cases. ${ }^{[33]}$ Various studies have not found pathogen in up to $40 \%-50 \%$ of children with presumed infectious diarrhea. ${ }^{[7,17]}$

\section{Conclusion}

This study presents the current epidemiological status of diarrheal agents in North India which highlights that Rotavirus and DEC appear to be major cause of diarrhea in young children followed by $V$. cholerae, S. flexneri, E. histolytica, and G. lamblia. E. coli O157:H7 is unlikely to be causative agent of diarrhea. Culture and PCR are more reliable tests than ELISA for detection of E. coli O157:H7. Most of the bacterial isolates from the stool specimens showed high level of resistance to first-line antimicrobial agents used for empirical treatment of diarrhea which is matter of concern. Laboratory monitoring of drug susceptibility of stool isolates appears necessary to formulate antibiotic policy for treating diarrheal illness at local level. In future, studies with larger sample size, wider coverage, and epidemiological surveillance are extremely essential to provide valuable insights to knowledge of etiology of childhood diarrhea.

\section{Financial support and sponsorship}

Nil.

\section{Conflicts of interest}

There are no conflicts of interest.

\section{References}

1. World Health Organization. Diarrheal Disease. Fact Sheet; Updated May, 2017. Available from: http://www.who. int/mediacentre/factsheets/fs330/en/. [Last accessed on 2018 Aug 03].

2. Raji MI, Ibrahim YK. Prevalence of waterborne infections in Northwest Nigeria: A retrospective study. J Public Health Epidemiol 2011;3:382-5.
3. Lakshminarayanan S, Jayalakshmy R. Diarrheal diseases among children in India: Current scenario and future perspectives. J Nat Sci Biol Med 2015;6:24-8.

4. Government of India. National Child Survival and Safe Motherhood Programme, MCH Division, Department of Family Welfare Ministry of Health and Family Welfare. New Delhi: Government of India; 2015.

5. Feleke H, Medhin G, Abebe A, Beyene B, Kloos H, Asrat D, et al. Enteric pathogens and associated risk factors among under-five children with and without diarrhea in Wegera district, Northwestern Ethiopia. Pan Afr Med J 2018;29:72.

6. Operario DJ, Platts-Mills JA, Nadan S, Page N, Seheri M, Mphahlele J, et al. Etiology of severe acute watery diarrhea in children in the global rotavirus surveillance network using quantitative polymerase chain reaction. J Infect Dis 2017;216:220-7.

7. Karmali MA. Emerging public health challenges of Shiga toxin-producing Escherichia coli related to changes in the pathogen, the population, and the environment. Clin Infect Dis 2017;64:371-6.

8. Samie A, Guerrant RL, Barrett L, Bessong PO, Igumbor EO, Obi CL, et al. Prevalence of intestinal parasitic and bacterial pathogens in diarrhoeal and non-diarroeal human stools from Vhembe district, South Africa. J Health Popul Nutr 2009;27:739-45.

9. Huang SH, Lin YF, Tsai MH, Yang S, Liao ML, Chao SW, et al. Detection of common diarrhea-causing pathogens in Northern Taiwan by multiplex polymerase chain reaction. Medicine (Baltimore) 2018;97:e11006.

10. Pal A, Ghosh S, Ramamurthy T, Yamasaki S, Tsukamoto T, Bhattacharya SK, et al. Shiga-toxin producing Escherichia coli from healthy cattle in a semi-urban community in Calcutta, India. Indian J Med Res 1999;110:83-5.

11. Saxena T, Kaushik P, Krishna Mohan M. Prevalence of E. coli O157:H7 in water sources: An overview on associated diseases, outbreaks and detection methods. Diagn Microbiol Infect Dis 2015;82:249-64.

12. Henriksen SA, Pohlenz JF. Staining of cryptosporidia by a modified Ziehl-Neelsen technique. Acta Vet Scand 1981;22:594-6.

13. March SB, Ratnam S. Sorbitol-MacConkey medium for detection of Escherichia coli O157:H7 associated with hemorrhagic colitis. J Clin Microbiol 1986;23:869-72.

14. CLSI. Performance Standards for Antimicrobial Susceptibility Testing; Twenty-Six Informational Supplement. CLSI document M100-S26. Wayne, PA: Clinical and Laboratory Standards Institute; 2016.

15. Aranda KR, Fagundes-Neto U, Scaletsky IC. Evaluation of multiplex PCRs for diagnosis of infection with diarrheagenic Escherichia coli and Shigella spp. J Clin Microbiol 2004;42:5849-53.

16. Hegde A, Ballal M, Shenoy S. Detection of diarrheagenic Escherichia coli by multiplex PCR. Indian J Med Microbiol 2012;30:279-84.

17. Panda A, Chandrakar SK, Bawankar S, Chandi DH. Etiology of bacterial and parasitological pediatric diarrhea in a tertiary care hospital. Asian J Med Sci 2015;7:54-7.

18. Chavan SK, Harshan KH. Aerobic bacteriological study with their antibiogram in children with acute diarrhea in North East Karnataka, India. Int J Curr Microbiol App Sci 2015;4:700-6.

19. Pol SS, Dedwal AK, Ranshing SS, Chitambar SD, Pednekar SN, Bharadwaj RS, et al. Prevalence and characterization of rotaviruses in children hospitalized for diarrheal disease in a tertiary care hospital, Pune. Indian J Med Microbiol 2017;35:33-6.

20. Ramani S, Kang G. Burden of disease \& molecular epidemiology of group A rotavirus infections in India. Indian J Med Res 2007;125:619-32.

21. Gazal S, Taku A, Bhat MA, Badroo G. Group A rotavirus and bacterial agents associated with diarrhoea-induced hospitalisations in children below 5 years of age in Jammu. Indian 
J Med Microbiol 2014;32:57-9.

22. Uma B, Prabhakar K, Rajendran S, Kavitha K, Sarayu YL. Antibiotic sensitivity and plasmid profiles of Escherichia coli isolated from pediatric diarrhea. J Glob Infect Dis 2009;1:107-10.

23. Becker SL, Chatigre JK, Gohou JP, Coulibaly JT, Leuppi R, Polman K, et al. Combined stool-based multiplex PCR and microscopy for enhanced pathogen detection in patients with persistent diarrhoea and asymptomatic controls from Côte d'ivoire. Clin Microbiol Infect 2015;21:591.e1-10.

24. Zhou Y, Zhu X, Hou H, Lu Y, Yu J, Mao L, et al. Characteristics of diarrheagenic Escherichia coli among children under 5 years of age with acute diarrhea: A hospital based study. BMC Infect Dis 2018;18:63.

25. Tian L, Zhu X, Chen Z, Liu W, Li S, Yu W, et al. Characteristics of bacterial pathogens associated with acute diarrhea in children under 5 years of age: A hospital-based cross-sectional study. BMC Infect Dis 2016;16:253.

26. Chen Y, Chen X, Zheng S, Yu F, Kong H, Yang Q, et al. Serotypes, genotypes and antimicrobial resistance patterns of human diarrhoeagenic Escherichia coli isolates circulating in Southeastern China. Clin Microbiol Infect 2014;20:52-8.

27. Ballal M, Baby, Chandran A, Mathew M, Shivananda PG.
Shigella and the fluorinated quinolones. Indian J Pathol Microbiol 1998;41:453-6.

28. Kaistha N, Mehta M, Gautam V, Gupta V. Outbreak of cholera in $\&$ around Chandigarh during two successive years $(2002,2003)$. Indian J Med Res 2005;122:404-7.

29. Brotman M, Giannella RA, Alm PF, Bauman M, Bennett AR, Black RE, et al. Consensus Conference statement: E coli 0157:H7 infections - an emerging national health crisis. Gastroenterology. 1995;108:1923-34.

30. Bettelheim KA, Evangelidis H, Pearce JL, Sowers E, Strockbine NA. Isolation of a Citrobacter freundii strain which carries the Escherichia coli O157 antigen. J Clin Microbiol 1993;31:760-1.

31. Jad B, Dogra S, Mahajan B. Significant decrease in prevalence of intestinal parasites among patients seeking treatment in a tertiary care hospital in Jammu: A changing trend. Int J Curr Microbiol App Sci 2015;4:659-4.

32. Dabas A, Shah D, Bhatnagar S, Lodha R. Epidemiology of Cryptosporidium in pediatric diarrheal illnesses. Indian Pediatr 2017;54:299-309.

33. Walker CLF, Rudan I, Liu L, Nair H, Theodoratou E, Bhutta ZA, et al. Global burden of childhood pneumonia and diarrhoea. Lancet 2013;381:1405-16. 